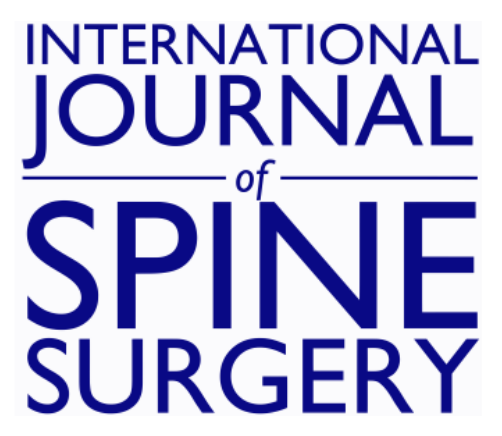

\title{
The Transverse Process Trajectory Technique: An Alternative for Thoracic Pedicle Screw Implantation--Radiographic and Biomechanical Analysis
}

Baron Lonner, Kushagra Verma, Chanland Roonprapunt, Yuan Ren, Casey A. Slattery, Ahmet Alanay, Gabrielle Kassin, Andrea Castillo, Mohamad Bazerbashi, Mark A. Buehler, Manoj K. Kodigudla, Amey V. Kelkar, Hassan Serhan and Vijay Goel

Int J Spine Surg 2021, 15 (2) 315-323

doi: https://doi.org/10.14444/8041

http://ijssurgery.com/content/15/2/315

This information is current as of April 25, 2023.

Email Alerts Receive free email-alerts when new articles cite this article. Sign up at:

http://ijssurgery.com/alerts

The International Journal of Spine Surgery

2397 Waterbury Circle, Suite 1,

Aurora, IL 60504, Phone: +1-630-375-1432 


\title{
The Transverse Process Trajectory Technique: An Alternative for Thoracic Pedicle Screw Implantation- Radiographic and Biomechanical Analysis
}

\author{
BARON LONNER, MD,${ }^{1}$ KUSHAGRA VERMA, MD, MS,${ }^{2}$ CHANLAND ROONPRAPUNT, MD, PHD, ${ }^{1}$ \\ YUAN REN, PHD, ${ }^{1}$ CASEY A. SLATTERY, MD,${ }^{3}$ AHMET ALANAY, MD,${ }^{4}$ GABRIELLE KASSIN, BS, ${ }^{1}$ \\ ANDREA CASTILLO, BS, ${ }^{1}$ MOHAMAD BAZERBASHI, MD,${ }^{5}$ MARK A. BUEHLER, MD,${ }^{5}$ MANOJ K. \\ KODIGUDLA, MS, ${ }^{5}$ AMEY V. KELKAR, MS, ${ }^{5}$ HASSAN SERHAN, PHD,${ }^{6}$ VIJAY GOEL, PHD ${ }^{5}$ \\ ${ }^{1}$ Mount Sinai Hospital, New York, New York, ${ }^{2}$ Verma Spine, Los Alamitos, California, ${ }^{3}$ University of New Mexico Hospital, Albuquerque, New Mexico, \\ ${ }^{4}$ Department of Orthopaedics and Traumatology, Acibadem University School of Medicine, Istanbul, Turkey, ${ }^{5}$ University of Toledo, Toledo, Ohio, ${ }^{6}$ DePuy Synthes \\ Spine, Inc, Raynham, Massachusetts
}

\begin{abstract}
Background: This study evaluates the accuracy, biomechanical profile, and learning curve of the transverse process trajectory technique (TPT) compared to the straightforward (SF) and in-out-in (IOI) techniques. SF and IOI have been used for fixation in the thoracic spine. Although widely used, there are associated learning curves and symptomatic pedicular breaches. We have found the transverse process to be a reproducible pathway into the pedicle.

Methods: Three surgeons with varying experience (experienced [E] with 20 years in practice, surgeon [S] with less than 10 years in practice, and senior resident trainee [T] with no experience with TPT) operated on 8 cadavers. In phase 1, each surgeon instrumented 2 cadavers, alternating between TPT and SF from T1 to T12 ( $\mathrm{n}=48$ total levels). In phase 2, the E and T surgeons instrumented 1 cadaver each, alternating between TPT and IOI. Computed tomography scans were analyzed for accuracy of screw placement, defined as the percentage of placements without critical breaches. Axial pullout and derotational force testing were performed. Statistical analyses include paired $t$ test and analysis of variance with Tukey correction.

Results: Overall accuracy of screw placement was comparable between techniques (TPT: 92.7\%; SF: 97.2\%; IOI: $95.8 \% ; P=.4151)$. Accuracy by technique did not differ for each individual surgeon $(\mathrm{E}: P=.7733 ; \mathrm{S}: P=.3475 ; \mathrm{T}: P=$ .4191 ) or by experience level by technique (TPT: $P=.1127$; FH: $P=.5979$; IOI: $P=.5935$ ). Pullout strength was comparable between TPT and SF (571 vs $442 \mathrm{~N}, P=.3164)$ but was greater for TPT versus IOI (454 vs $215 \mathrm{~N}, P=$ .0156). There was a trend toward improved derotational force for TPT versus SF (1.06 vs $0.93 \mathrm{Nm} / \mathrm{degrees}, P=.0728$ ) but not for TPT versus IOI (1.36 vs $1.16 \mathrm{Nm}$ /degrees, $P=.74$ ). Screw placement time was shortest for E and longest for $\mathrm{T}$ for TPT and SF and not different for IOI (TPT: $P=.0349$; SF: $P<.0001$; IOI: $P=.1787$ ) but did not vary by technique.
\end{abstract}

Conclusions: We describe the TPT, which uses the transverse process as a corridor through the pedicle. TPT is an accurate method of thoracic pedicle screw placement with potential biomechanical advantages and with acceptable learning curve characteristics.

Clinical Relevance: This study provides the surgeon with a new trajectory for pedicle screw placement that can be used in clinical practice.

\section{Biomechanics}

Keywords: thoracic pedicle screw implantation, transverse process trajectory technique, instrumentation, thoracic spinal deformities, biomechanical characteristics, screw placement, accuracy of placement, reproducibility, spine surgeon learning curve

\section{INTRODUCTION}

Current instrumentation standards for correction of scoliosis and other thoracic spinal deformities include the use of thoracic pedicle screws. ${ }^{1,2}$ The anatomy of the pedicles in normal and scoliotic spines has been studied and serves as the basis for pedicle screw insertion techniques. ${ }^{3-5}$ A number of techniques to facilitate accurate placement of pedicle screws have been used, including the straightforward (SF) technique, funnel, in-out-in (IOI) techniques (Figure 1), fluoroscopically assisted screw placement, lamino-foraminal pedicle palpation, and various other forms of intraoperative 


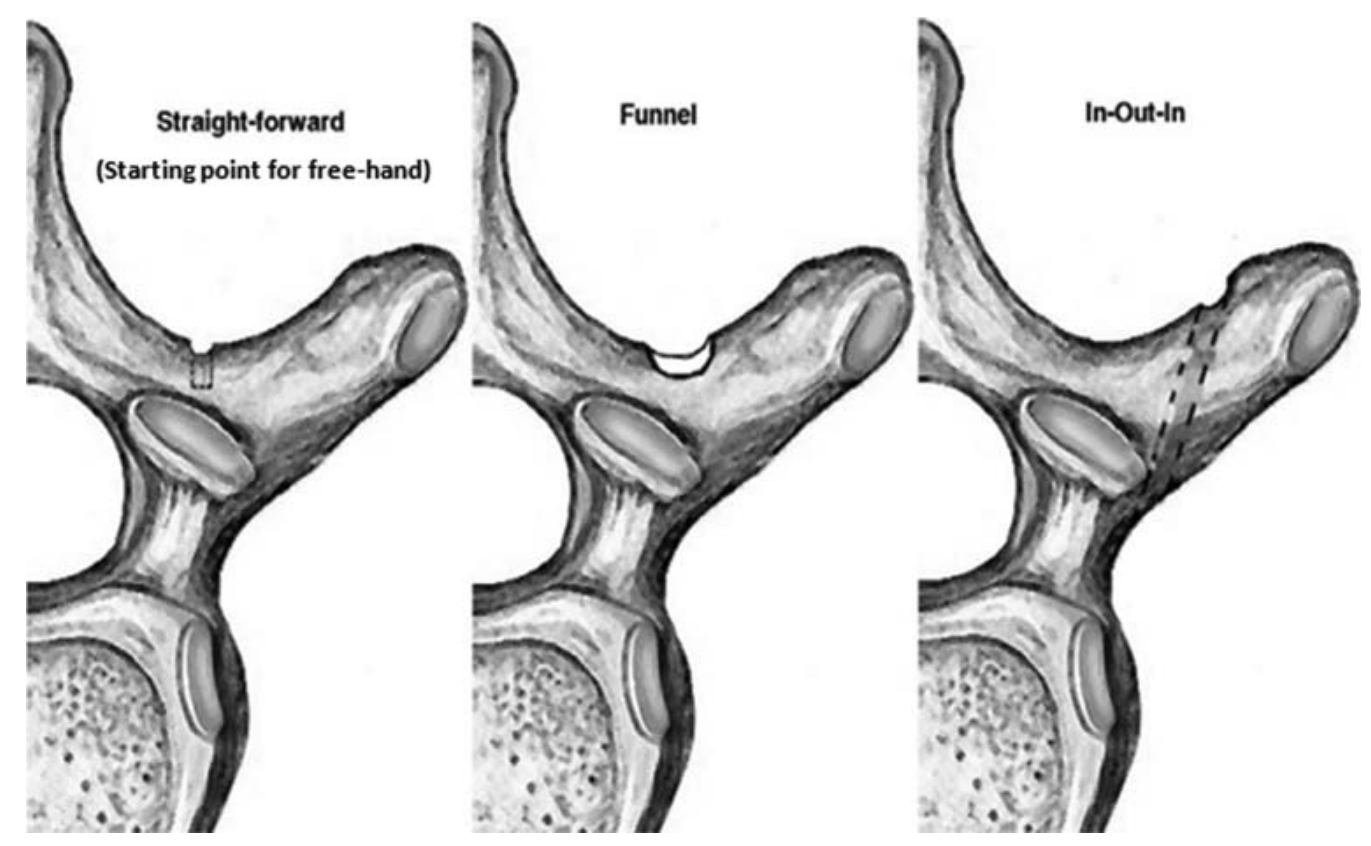

Figure 1. Starting points for straightforward, funnel, and in-out-in techniques.

navigation. ${ }^{6-12}$ Figure 1 also shows that the rib facet on the tip of the transverse process (TP) forces the entrance point into the trajectory to be more medial. This anatomical fact is taken into account in order to avoid canal breach through the described trajectories. Each technique has its proponents, the goal being accurate and efficient placement and minimization of neurological deficits and reoperation for errant screw placement. ${ }^{13-16}$ Breaches of the thoracic pedicle following the placement of pedicle screws have been reported to range from $2 \%$ to $15.7 \% .{ }^{17,18}$ The accuracy of placement has been shown to be impacted by the level of the surgeon's and the assistant's experience. ${ }^{19,20}$

Our team has modified its technique for screw placement over the years from a lamino-foraminal palpation-assisted approach to SF based on ana-

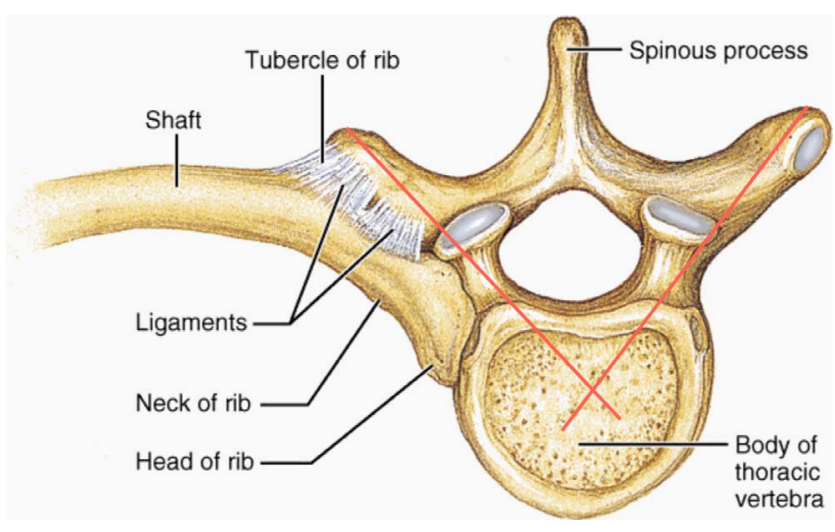

Figure 2. Trajectory for the transverse process trajectory technique in red. tomical landmarks using a probe ${ }^{3}$ and to the use of a power drill in a standard transpedicular trajectory. It became clear that a lateral starting point at the tip of the TP is highly reproducible for accurately cannulating the thoracic pedicle ${ }^{21}$ (Figure 2). The start point is at the cephalad $1 / 3$ of the TP at its tip, using the TP as a guide for the trajectory. Drilling of the cortex is done primarily, followed by the introduction of a probe and a palpating instrument to ensure intact bony margins followed by placement of the screw. This is termed the TP trajectory technique (TPT) (Figure 3). We found that TPT facilitates screw placement in the scoliotic, rotated spine by using the TP trajectory, which serves as a corridor through the pedicle into the vertebral body. Previous study has shown the dimensions of the transverse process to be sufficient for placement of screws. $^{22}$ However, the TP is used solely as a trajectory for navigating the placement of the pedicle screw. Prior to placement of the screw, the TP is essentially removed down to the level of the intirotus of the pedicle. It differs from IOI in that it starts at the very tip of the TP and remains within bone its entire course. In contrast, the IOI starts at the junction of the middle and lateral thirds and leaves the TP, traverses the costo-transverse-vertebral space, and reenters the vertebral body (Figure $3)$.

The principal goal of this study is to describe TPT and report its reproducibility, accuracy, biomechanical characteristics, and variability in the hands of 
B)
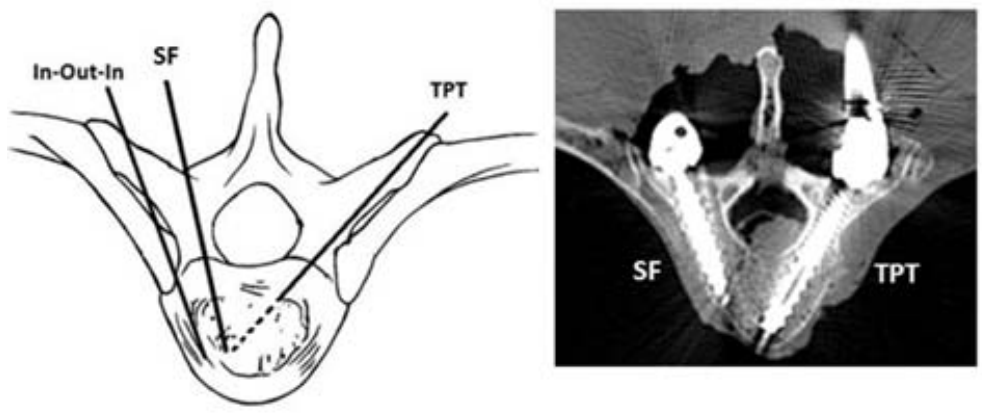

C)

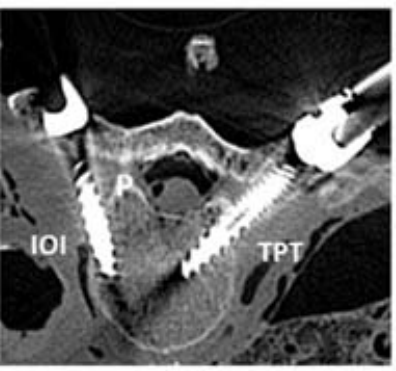

D)

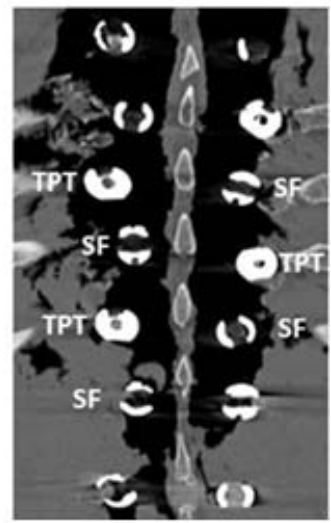

E)
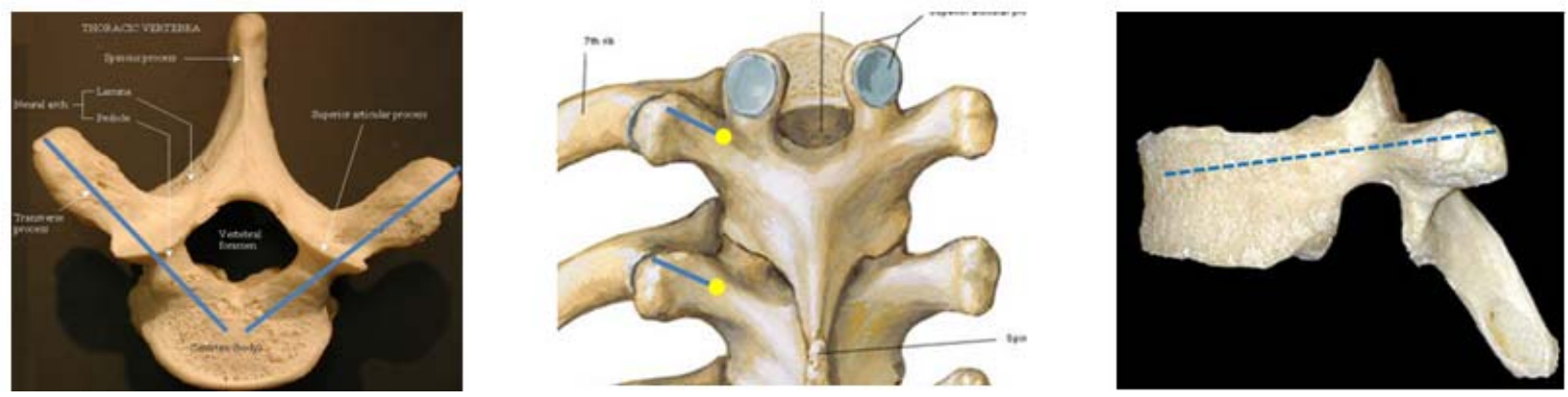

Figure 3. Illustration of (a) transverse process trajectory (TPT), straightforward (SF), and in-out-in (IOI) techniques; (b) axial CT cuts comparing TPT and SF and; (c) IOI and TPT trajectories; (d) coronal CT comparing SF and TPT; and (e) TPT trajectory in axial, coronal, and sagittal planes.

an experienced spine surgeon (20 years of experience), spine surgeon with less than 10 years of experience, and a resident/fellow trainee with no experience with TPT. The technique will be compared to more commonly performed SF and IOI with the expectation that accuracy will be improved with greater surgeon experience and familiarity with the technique.

Our hypotheses were as follows:

1. TPT will be as accurate as SF and IOI in terms of cannulating the vertebral pedicle without critical breaches.

2. TPT will provide comparable pullout to the traditional SF. Compared to IOI, TPT will likely provide improved pullout due to the longer lever arm associated with the technique and increased bony purchase.

3. TPT will have improved derotational strength compared to $\mathrm{SF}$ and IOI due to the more lateral starting point.

Efficiency of screw placement will be equivalent between the 3 surgeons of varying experience level but will be decreased for the TPT and IOI due to a lack of familiarity with these techniques.

\section{METHODS}

\section{Phases 1 and 2}

Eight fresh frozen human cadaveric torsos were procured for this study: 6 in phase 1 and 2 in phase 2 . The specimens were screened with radiographs to rule out preexisting deformities, fractures, or tumors. Three surgeons with varying experience participated in the study (experienced [E] with 20 years in practice, surgeon [S] with less than 10 years in practice, senior resident trainee $[\mathrm{T}]$ with no experience with TPT). Demographic data for the cadaver specimens were not available. Standard surgical exposure was used in the laboratory.

In phase 1 of this study, each surgeon instrumented the thoracic levels for 2 cadaveric torsos (T1-T12, $\mathrm{n}=48$ total levels) using both TPT and SF. Two different vendors were used for the polyaxial favored-angled titanium screws. Brand 1 was used for TPT, while brand 2 was used for SF. 
No differences in screw size, design, or material were noted between brands 1 and 2, which were donated implants. On each side, we alternated between SF and TPT from T1 to T12. On the first cadaver, the surgeon placed the first screw beginning at T12 by $\mathrm{SF}$, the second screw at T10 and T8, and so on. On the contralateral side, the first screw was placed by TPT at T12 followed by T10 and T8 and so on. In this way, each of the even-numbered vertebral bodies was cannulated by both techniques. After this stage, the odd-numbered vertebral bodies were cannulated on the first side by TPT followed by SF on the contralateral side.

This alternation of techniques in each level minimized the problem of comparing the techniques in separate cadavers whose pedicle size, anatomy, and bone density were likely variable. In the second cadaver, the order of the technique first performed was reversed for the 2 sides so that the T12 screw was performed by TPT and the T11 screw by SF. This process was reversed for the contralateral side so that every vertebra level was instrumented with both SF and TPT screws.

In phase 2, the $\mathrm{E}$ and $\mathrm{T}$ surgeons were available, and each instrumented 1 cadaver. These 2 surgeons alternated between TPT and IOI just as in phase 1 . For both phases, the time was recorded from pilothole creation to final placement of each screw. We evaluated the variability in screw placement time between surgeons and overall time for each technique in terms of familiarity with the technique as well as between techniques for all surgeons. The implantation sequence, pedicle screw sizes, and implantation method were kept consistent across specimens.

\section{Radiographic and Biomechanical Analysis}

After instrumentation, a computed tomography scan identified the direction and magnitude of pedicle breaches using the Gertzbein scale. ${ }^{23}$ After radiographic analysis, each vertebral level was individually harvested and potted in polyester resin in preparation for biomechanical evaluation and visual inspection. Measurements of the lever arm (VB center of rotation to rod) for TPT were compared to SF and IOI.

Half of the specimens were used for axial pullout testing along the axis of the screws (Figure 4A), and the remaining specimens were subjected to derotational force application perpendicular to each screw axis (Figure 4B).

\section{Pullout Testing}

Each potted specimen was gripped in a 3-axes vise fixed on an XY table attached to an MTS test system (MTS Systems Corporation, Eden Prairie, Minnesota) load cell. The 3-axes vise was adjusted until the actuator and screw were coaxial. A loading fixture attached to the actuator was attached to the screw. Axial load was applied by moving the actuator under displacement control. Load displacement data were collected. Maximum force required to pull the screw out was computed.

\section{Derotational Testing}

Each potted specimen was gripped in an angular vise (A) mounted on a fixture. The fixture was gripped between the jaws of a 3-axes vise (B) fixed on an XY table attached to an MTS load cell. Vises $\mathrm{A}$ and $\mathrm{B}$ were adjusted until the actuator and screw axis were perpendicular. A loading fixture fixed to the actuator was attached to the extended sleeve of the screw. Axial load was applied by moving the actuator under displacement control at a rate of 30 $\mathrm{mm} / \mathrm{min}$. The load was applied from the lateral side for the left pedicle screw and the medial side for the right pedicle screw. Load (F) and displacement (D) data were collected.

Angle $(\theta)$ for the corresponding displacement of the actuator and moment were computed using the above formulas. Angle ( $\theta$, degrees) versus moment $(\mathrm{Nm})$ graphs were plotted. The slope of the plot, rotational stiffness ( $\mathrm{Nm} /$ degrees), was computed.

No image guidance or other confirmatory techniques were used for screw placement. The technique was familiar to the 3 individuals who performed the study, including the experienced surgeon, who developed it. The $\mathrm{S}$ surgeon had some clinical experience, assisting in approximately 10 cases in which TPT was used prior to the study. The $\mathrm{S}$ surgeon placed primarily convex pedicle screws, using TPT under the supervision of the E surgeon. The resident trainee had seen TPT performed months earlier in a cadaver course. Prior to instrumenting the cadavers with TPT, both of the less experienced surgeons were given a brief verbal tutorial on the technique. After this orientation, the senior surgeon did not oversee the placement of the screws.

Each screw was categorized in a dichotomous manner as breach or no breach along with the breach magnitude. Each breach was scored as a 
A)



B)
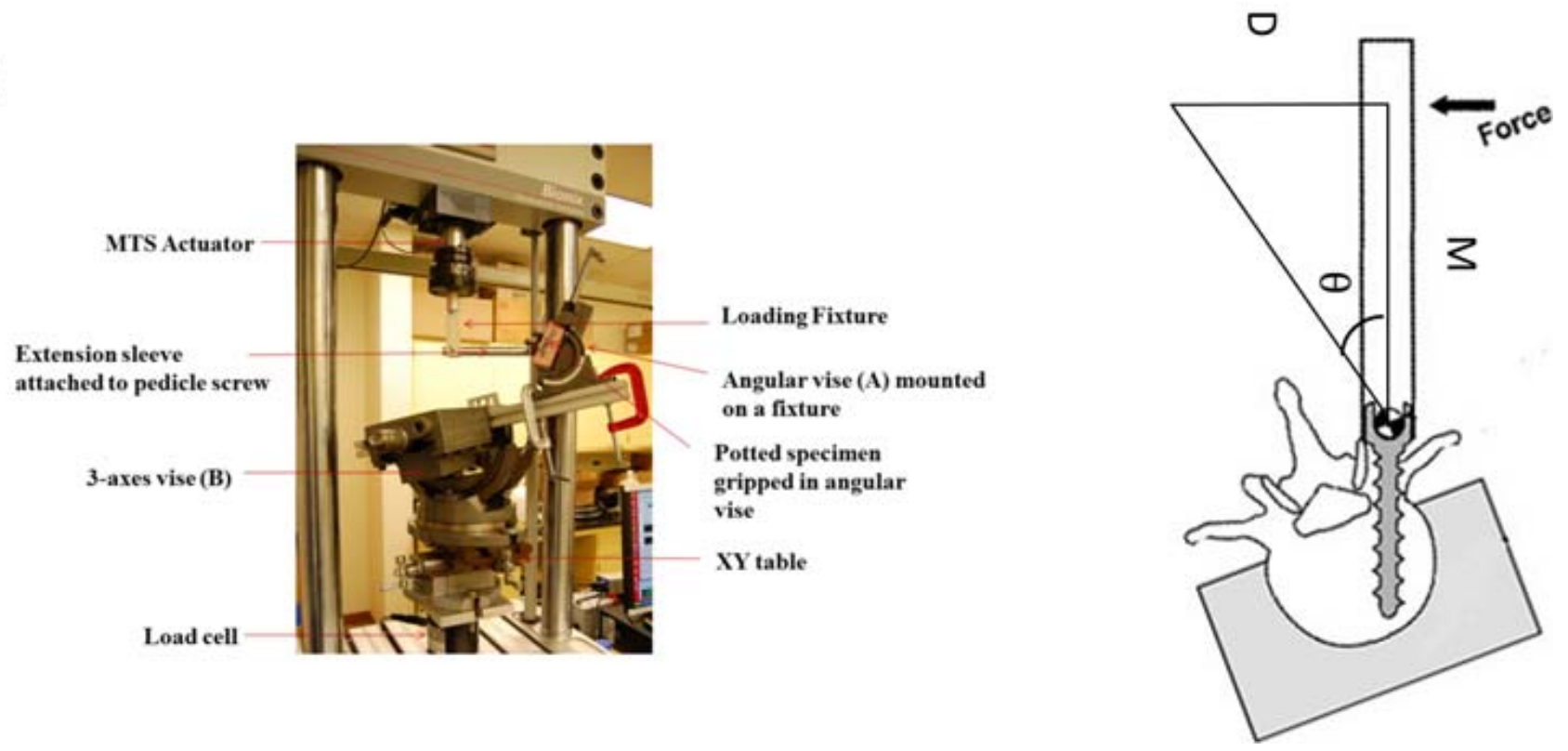

Figure 4. Photograph of an MTS actuator set-up for testing (a) axial pull-out strength and (b) rotational forces.

$<2-$, 2-4-, or $>4$-mm perforation. These breach rates were scored by a single graduate research assistant who was trained by a radiologist to measure breach rates. As a method of standardization, medial or caudal breaches $>4 \mathrm{~mm}$ were defined as critical. Accuracy of screw placement was reported as the percentage of total screws accurately placed without critical breach. In addition, the direction of the breach was recorded.

\section{Statistical Analysis}

A chi-square test compared the rate of perforations among the 3 surgeons for each technique. Fisher exact tests also compared the differences in the number of critical breaches per group of instrumented levels (T1-T5, T6-T10, T11-T12). A $t$ test (Wilcoxon rank sum test) was used to compare time spent on each screw placement for the 3 techniques. Analysis of variance with Bonferroni correction was used for efficiency comparisons between surgeons. The pullout and derotational strength of TPT, SF, IOI pedicle screws were quantified and statistically analyzed using a $t$ test and analysis of variance. Post hoc power analysis was performed to determine the power of the study using PASS 15.0 (NCSS, LLC, Kaysville, Utah).

\section{RESULTS}

The accuracy of screw placement between techniques was comparable (TPT: 92.7\%; SF: 97.2\%; IOI: $95.8 \% ; P=.415)$ and did not differ between surgeons $(\mathrm{E}: P=.773 ; \mathrm{S}: P=.348 ; \mathrm{T}: P=.419)$, as shown in Table 1. There was also no difference 
Table 1. Accuracy (percentage of total screws accurately placed without critical breach) of screw placement between the 3 surgeons.

\begin{tabular}{lcccc}
\hline & $\begin{array}{c}\text { TPT } \\
(\%)\end{array}$ & $\begin{array}{c}\text { SF } \\
(\%)\end{array}$ & $\begin{array}{c}\text { IOI } \\
(\%)\end{array}$ & $\begin{array}{c}\text { Within Surgeons } \\
(\boldsymbol{P} \text { Value })\end{array}$ \\
\hline Experienced surgeon & 97.2 & 95.8 & 100.0 & .773 \\
Surgeon & 83.3 & 95.8 & NA & .348 \\
Resident trainee & 94.4 & 100.0 & 91.7 & .419 \\
Between surgeons $(P$ value $)$ & .113 & .598 & .594 & \\
\hline
\end{tabular}

Abbreviations: IOI, in-out-in; NA, not applicable; SF, straightforward; TPT,

transverse process trajectory technique.

when evaluating the surgeons by experience level per technique (TPT: $P=.113$; SF: $P=.598$; IOI: $P=$ $.594)$.

In phase 1, all critical breaches by TPT were medial. For SF, 1 critical breach was medial for the E surgeon and 1 was caudal for the $\mathrm{S}$ surgeon. Of all lateral breaches, most were associated with the SF versus TPT (70\% vs $7.7 \% ; P=.0009)$. However, noncritical medial breaches were more common for TPT, comprising $92.3 \%$ of all noncritical breaches for this technique compared to only $15 \%$ for SF ( $P$ $<.0001)$. There were no differences in the number of critical breaches per group of levels $(P=.461)$ and no significant differences in the number of critical breaches per level $(P=.389)$.

Biomechanical force analysis is shown in Table 2. Axial pullout strength was comparable between TPT and SF (TPT: $571 \mathrm{~N}$; SF: $442 \mathrm{~N} ; P=.316$ ) but was greater for TPT versus IOI in the phase 2 analysis (TPT: $454 \mathrm{~N}$; IOI: $215 \mathrm{~N} ; P=.0156$ ). There was a trend toward improved derotational force by TPT compared SF (TPT: 1.07 Nm/degrees; SF: 0.97 $\mathrm{Nm} /$ degrees; $P=.073$ ) but not for TPT versus IOI (TPT: $1.36 \mathrm{Nm} /$ degrees; IOI: $1.23 \mathrm{Nm} /$ degrees; $P=$ .74). These comparisons were performed independently and therefore do not have a direct comparison of SF versus IOI. The differences observed were not statistically significant.

Comparing the 2 techniques, there was no difference in the screw placement times in phase 1 for both the $\mathrm{E}$ and $\mathrm{T}$ surgeons for TPT versus SF (E: 1.32 vs 1.36 minutes; $P=.8093 ; \mathrm{T}: 2.43$ vs 2.27 minutes; $P=.6389$ ). The $\mathrm{S}$ surgeon took slightly longer to place TPT versus SF (TPT: 1.75 minutes; SF: 1.43 minutes; $P=.0331)$. Evaluating efficiency times for phases 1 and 2 (Table 3), the resident was the slowest among the 3 surgeons in screw placement using TPT and SF (TPT: $P=.0349$; SF: $P<.0001)$. However, in phase 2, there was no difference between the $\mathrm{E}$ and $\mathrm{T}$ surgeons using IOI $(P=.18)$.
Table 2. Biomechanical forces for phases 1 and 2 .

\begin{tabular}{lcc}
\hline & Pullout (Nm/Degrees) & Derotational (Nm/Degrees) \\
\hline Phase 1 & & \\
TPT & 571 & 1.07 \\
SF & 442 & 0.97 \\
$P$ value & .316 & .073 \\
Phase 2 & & \\
TPT & 454 & 1.36 \\
IOI & 215 & 1.23 \\
$P$ value & .0156 & .74
\end{tabular}

Abbreviations: IOI, in-out-in; SF, straightforward; TPT, transverse process trajectory technique.

\section{DISCUSSION}

Correction of spinal deformities and anchorage to the thoracic spine for the treatment of various pathologies largely relies on pedicle screw instrumentation. Improved correction of spinal deformities, less loss of fixation, and reliable outcomes have been achieved for adolescent idiopathic scoliosis (AIS) and other entities with the use of pedicle screws rather than hooks or hybrid hook-screw constructs. $^{1,2,7}$ Various techniques for placement have been used that follow different trajectories into the pedicle. SF can direct a screw in an orthogonal or anatomical trajectory, while IOI takes advantage of the relatively safe costo-transverse and costovertebral corridor. This is particularly useful for small pedicles; however, the pullout strength testing characteristics have been shown to be less than the more common SF technique. ${ }^{24}$

Despite an abundance of literature pointing to the benefits of this fixation in the treatment of spinal deformities, screw misplacement occurs in $2 \%-15 \%$ of cases. ${ }^{6,25}$ The scoliotic spine in particular poses challenges for surgeons, as pedicles are often dysplastic and rotated. The TP may also be deformed, though to our knowledge this has not been shown in an anatomic article. This occurs particularly on the concave curvature, with rotation of the curve apex requiring adjustments of the medio-lateral screw trajectory, which can be difficult to judge clinically without the assistance of cumbersome image guidance systems. ${ }^{26}$ Furthermore, the value of image guidance systems is unproven due to a lack of large randomized control trials looking at screw-related complications and breach rates. ${ }^{27}$ Some breaches are asymptomatic, while others cause neurological complications and the need for reoperation. ${ }^{1,2,27}$ Reoperation results in a traumatic experience to the patient, may not result in full resolution of symptoms, and adds cost. ${ }^{27}$ 
Table 3. Efficiency of screw placement among the 3 surgeons.

\begin{tabular}{|c|c|c|c|c|}
\hline & TPT (min) & SF (min) & IOI (min) & $P$ Value \\
\hline Experienced surgeon & 1.44 & 1.36 & 1.78 & .207 \\
\hline Surgeon & 1.75 & 1.43 & NA & .033 \\
\hline Resident trainee & 2.37 & 2.27 & 1.68 & .179 \\
\hline \multirow[t]{2}{*}{ Between surgeons } & $P=.0349$ & $P<.0001$ & $P=.18$ & \\
\hline & $\begin{array}{l}\text { Resident slowest; no difference between } \\
\text { experienced surgeon and surgeon }\end{array}$ & $\begin{array}{l}\text { Resident slowest; no difference between } \\
\text { experienced surgeon and surgeon }\end{array}$ & No difference & \\
\hline
\end{tabular}

Abbreviations: IOI, in-out-in; NA, not applicable; SF, straightforward; TPT, transverse process trajectory technique.

Another component of accuracy and efficiency of pedicle screw placement is the learning curve that has been described for thoracic pedicle screw placement. This may impact the safety of the procedure as well as operative time, having a direct impact on blood loss, possible transfusions, and infection. ${ }^{8,28,29}$ Future study could include in vitro cadaver and/or virtual simulations to assess and overcome the learning curve.

Over the course of doing hundreds of cases and training residents/fellows, the TP was a reliable landmark following the trajectory of the pedicle. Through an iterative process, this technique was developed first using the standard freehand technique entrance point into the pedicle while using the TP as a visual guide to medio-lateral and cephalocaudal orientation. Later, the technique was modified to create a trough into the cephalad one-third of the TP and, following that, directly into the pedicle using a drill. Although we have found the technique to be clinically reliable, the purpose of this study was to objectively study the anatomic veracity, accuracy, and potential biomechanical advantages. We aimed for a worst-case scenario with no image guidance, senior surgeon oversight, electromyography, or spinal cord monitoring.

Our data show comparable accuracy rates: $92.7 \%$ for TPT, $97.2 \%$ for SF, and $95.8 \%$ for IOI. Post hoc power analysis indicated that our study $(\mathrm{n}=48)$ was not adequately powered to conclude that there is no difference in accuracy of screw placement using TPT versus SF $(\alpha=0.05)$. However, accuracy was similar for all techniques for both the experienced surgeon and the resident trainee although less so for the less experienced surgeon. These differences may reflect a learning curve of the technique that must be respected. Newer technical innovations, such as the use of spinal navigation, can mitigate any increase in breach rates that a surgeon in practice might experience for any of the techniques studied. By the nature of its trajectory, there is a greater possibility of medial breaches compared to SF and IOI, but we found this to be relatively low in this worst-case scenario. In addition, the technique may be used as a salvage technique when the traditional SF technique is not successful.

The biomechanical characteristics of TPT were not unexpected. Axial pullout strength, a fundamental test of adequacy of fixation, showed no significant difference between TPT and SF. Both techniques consist of a pedicle screw being in bone throughout its course. We did not test the potential benefits of placing longer screws that are afforded a more lateral starting point for the TPT and may result in improved pullout strength. ${ }^{31}$ Pullout strength was greater for TPT compared to IOI as expected given prior research showing improved pullout of SF versus IOI. ${ }^{24}$ Derotational forces were greater for TPT than for SF, but this did not achieve statistical significance. Comparing TPT to IOI, we expected improved derotational strength for TPT given the more lateral starting point and greater lever arm compared to the other techniques. Perhaps a clearer advantage would be found had longer screws been used for the TPT. This latter biomechanical advantage may prove beneficial in axial plane correction of scoliosis.

Although this article does not directly show the learning curve of the technique, we do report the accuracy with various levels of experience. There seems to be comparable accuracy rates found for surgeons of all levels of experience. Both the critical and the noncritical breach rates did not differ significantly for any of the surgeons, although the $\mathrm{S}$ surgeon had a larger discrepancy with more critical breaches by TPT than by SF. There was a greater tendency for medial breaches to occur for TPT versus SF as outlined above.

Screw placement time was greater for the $T$ surgeon as expected compared to the E surgeon for TPT and SF but not IOI. However, the efficiency of screw placement did not differ between techniques for all surgeons. The trainee had experience with SF 
both in clinical residency/fellowship and in cadaver labs over the years of training.

In summary, TPT can be used as a primary technique or salvage for difficult screw placement and can be alternated with SF and IOI as long as favor-angled screws are used, making it a highly versatile screw placement technique. It may be beneficial in educating resident and fellowship trainees by taking advantage of the TP trajectory as a guide into the pedicle without expensive and cumbersome image guidance techniques. ${ }^{30-35}$

\section{CONCLUSIONS}

We report a new technique for placement of thoracic pedicle screws that uses the TP as the corridor for pedicle entry, the TPT. In this study involving 3 surgeons of different experience levels and 8 cadavers, there were no significant differences in critical breaches of the pedicle between TPT, SF, and IOI. The TPT offers a reproducible method of pedicle screw placement with similar pullout and derotational strength as established techniques. This new technique has similar accuracy, efficiency and biomechanical profiles as the established SF and IOI. Future approaches should include training and in vitro simulations to overcome the learning curve.

\section{REFERENCES}

1. Suk SI, Lee CK, Kim WJ, Chung YJ, Park YB. Segmental pedicle screw fixation in the treatment of thoracic idiopathic scoliosis. Spine. 1995;20(12):1399-1405.

2. Kim YJ, Lenke LG. Thoracic pedicle screw placement: free-hand technique. Neurol India. 2005;53(4):512-519.

3. Zindrick MR, Wiltse LL, Doornik A, et al. Analysis of the morphometric characteristics of the thoracic and lumbar pedicles. Spine (Phila Pa 1976). 1987;12(2):160-166.

4. Vaccaro AR, Rizzolo SJ, Allardyce TJ, et al. Placement of pedicle screws in the thoracic spine. Part I: morphometric analysis of the thoracic vertebrae. $J$ Bone Jt Surg Am. 1995;77(8):1193-1199.

5. Liljenqvist UR, Allkemper T, Hackenberg L, Link TM, Steinbeck J, Halm HF. Analysis of vertebral morphology in idiopathic scoliosis with use of magnetic resonance imaging and multiplanar reconstruction. J Bone Jt Surg Am. 2002;84A(3):359-368.

6. Kim YJ, Lenke LG, Bridwell KH, Cho YS, Riew KD. Free hand pedicle screw placement in the thoracic spine: is it safe? Spine (Phila Pa 1976). 2004;29(3):333-342; discussion 342 .

7. Lonner BS, Auerbach JD, Estreicher MB, Kean KE. Thoracic pedicle screw instrumentation: the learning curve and evolution in technique in the treatment of adolescent idiopathic scoliosis. Spine (Phila Pa 1976). 2009;34(20):2158-2164.

8. Zeiller SC, Lee J, Lim M, Vaccaro AR. Posterior thoracic segmental pedicle screw instrumentation: evolving methods of safe and effective placement. Neurol India. 2005;53(4):458-465

9. Shin BJ, James AR, Njoku IU, Hartl R. Pedicle screw navigation: a systematic review and meta-analysis of perforation risk for computer-navigated versus freehand insertion. $J$ Neurosurg Spine. 2012;17(2):113-122.

10. Hu X, Ohnmeiss DD, Lieberman IH. Robotic-assisted pedicle screw placement: lessons learned from the first 102 patients. Eur Spine J. 2013;22(3):661-666.

11. Belmont PJ Jr, Klemme WR, Dhawan A, Polly DW Jr. In vivo accuracy of thoracic pedicle screws. Spine. 2001;26(21):2340-2346.

12. Gaines RW Jr. The use of pedicle-screw internal fixation for the operative treatment of spinal disorders. J Bone Jt Surg Am. 2000;82-A(10):1458-1476.

13. Samdani AF, Belin EJ, Bennett JT, et al. Unplanned return to the operating room in patients with adolescent idiopathic scoliosis. Spine. 2013;38(21):1842-1847.

14. Kim YJ, Lenke LG. Review article: thoracic pedicle screw placement: free-hand technique. Neurol India. December 2005;53(4):512-519.

15. Belmont PJ Jr, Klemme WR, Robinson M, Polly DW. Accuracy of thoracic pedicle screws in patients with and without coronal plane spinal deformities. Spine. 2002;27(14):1558-1566.

16. Polly DW, Potter BK, Kuklo T, Young S, Johnson C, Klemme WR. Volumetric spinal canal intrusion: a comparison between thoracic pedicle screws and thoracic hooks. Spine (Phila Pa 1976). 2004;29(1):63-69.

17. Gertzbein SD, Robbins SE. Accuracy of pedicular screw placement in vivo. Spine (Phila Pa 1976). 1990;15(1):11-14.

18. Wollowick A, Sarwahi V, Horn J, et al. burying one's head in the sand: are we underestimating the significance of pedicle screw misplacement? Spine J. 2011;11(10):S9-S10.

19. Cahill PJ, Samdani AF, Pahys JM, et al. Youth and experience: the effect of surgeon experience on outcomes in AIS surgery: paper \#45. Spine Journal Meeting Abstracts: 2011. p 78.

20. Auerbach J, Lonner B, Antonacci D, Kean K. Is the experience level of the surgical assistant in scoliosis surgery a risk factor for perioperative complications? Poster presented at: North American Spine Society (NASS), October 23-27, 2007, Austin, TX.

21. Heller JG, Shuster JK, Hutton WC. Pedicle and transverse process screws of the upper thoracic spine: biomechanical comparison of loads to failure. Spine. 1999;24(7):654 658.

22. Cui X, Cai J, Sun J, Jian Z. Morphology study of thoracic transverse processes and its significance in pedicle-rib unit screw fixation. J Spinal Disord Tech. 2015;28(2):E74-E77.

23. Gertzbein SD, Robbins SE. Accuracy of pedicular screw placement in vivo. Spine (Phila Pa 1976). 1990;15:11-14.

24. Brown BS, McIff TE, Glattes RC, Burton DC, Asher MA. The effect of starting point placement technique on thoracic transverse process strength: an ex vivo biomechanical study. Scoliosis. 2010;13;5:14. doi:10.1186/1748-7161-5-14

25. Yi-Jiang H, Peng M-X, He S-Q, Liu L-L, Dai M-H, Tang C. Biomechanical study of the funnel technique applied in thoracic pedicle screw replacement. Afr Health Sci. 2014;14(3):716-724.

26. Lehman RA Jr, Lenke LG, Keeler KA, Kim YJ, Cheh G. Computed tomography evaluation of pedicle screws placed 
in the pediatric deformed spine over an 8-year period. Spine (Phila Pa 1976). 2007;32(24):2679-2684.

27. Chan A, Parent E, Narvacan K, San C, Lou E. Intraoperative image guidance compared with free-hand methods in adolescent idiopathic scoliosis posterior spinal surgery: a systematic review on screw-related complications and breach rates. Spine J. 2017;17(9):1215-1229.

28. Larson AN, Polly DW Jr, Ackerman SJ, et al. Minimize Implants Maximize Outcomes Study Group. What would be the annual cost savings if fewer screws were used in adolescent idiopathic scoliosis treatment in the US? J Neurosurg Spine. 2016;24(1):116-123. doi:10.3171/2015.4.SPINE131119.

29. Lonner BS, Scharf C, Antonacci D, Goldstein Y, Panagopoulos G. The learning curve associated with thoracoscopic spinal instrumentation. Spine (Phila Pa 1976). 2005;30(24):2835-2840.

30. Lonner BS, Ren Y, Yaszay B, Kassin GM, Newton PO. Can surgical innovation decrease operative time, surgeon variability and surgical morbidity in AIS surgery: a study of operative efficiency ePoster with defense time. Paper presented at: North American Spine Society 31st Annual Meeting (NASS); October 26-29, 2016; Boston, MA.

31. Christodoulou E, Chinthakunta S, Reddy D, et al. Axial pullout strength comparison of different screw designs: fenestrated screw, dual outer diameter screw and standard pedicle screw. Scoliosis. 2015;10:15. doi:10.1186/s13013-0150039-6

32. Kotani T, Akazawa T, Sakuma T, et al. Accuracy of pedicle screw placement in scoliosis surgery: a comparison between conventional computed tomography-based and $\mathrm{O}-$ arm-based navigation techniques. Asian Spine J. 2014;8(3):331338. doi:10.4184/asj.2014.8.3.331

33. Sembrano JN, Polly DW Jr, Ledonio CGT, Santos ERG. Intraoperative 3-dimensional imaging (O-arm) for assessment of pedicle screw position: does it prevent unacceptable screw placement? Int J Spine Surg. 2012;6:49-54. doi:10. 1016/j.ijsp.2011.11.002
34. Flynn JM, Sakai DS. Improving safety in spinal deformity surgery: advances in navigation and neurologic monitoring. Eur Spine J. 2013;22(suppl 2):131-137. doi:10. 1007/s00586-012-2360-6

35. Ughwanogho E, Patel NM, Baldwin KD, Sampson NR, Flynn J. Computed tomography-guided navigation of thoracic pedicle screws for AIS results in more accurate placement and less screw removal. Spine. 2012;37(8):E473-E478.

Disclosures and COI: Research grant funding was received by DePuy Synthes Spine, Inc. The work was supported in part by the NSF Industry/ University Cooperative Research Center at the University of California at San Francisco, San Francisco, California, and the University of Toledo, Toledo, Ohio. Institutional review board approval was obtained for this study from all participating sites.

Corresponding Author: Baron Lonner, MD, Professor of Orthopaedic Surgery, Mount Sinai Hospital, 5 E $98^{\text {th }}$ Street, $4^{\text {th }}$ Floor, New York, NY 10029. Phone: (212) 241 8312; Fax: (646)537 9449; Email: baron.lonner@mountsinai.org.

Published 16 April 2021

This manuscript is generously published free of charge by ISASS, the International Society for the Advancement of Spine Surgery. Copyright (C) 2021 ISASS. To see more or order reprints or permissions, see http://ijssurgery.com. 